\title{
Going Beyond Blockchain Adoption's Hype to Improve Supply Chain Sustainability: Evidence From Empirical and Modelling Studies
}

\author{
Tinhinane Chikhi \\ Business School \\ Université de Sherbrooke \\ tinhinane.chikhi@usherbrooke.ca
}

\author{
Lucas Antonio Risso \\ Department of Industrial Engineering \\ Federal University of São Carlos \\ lucasrisso@gmail.com
}

\author{
Luis Antonio De Santa-Eulalia \\ Business School \\ Université de Sherbrooke \\ 1.santa-eulalia@usherbrooke.ca
}

\author{
Moacir Godinho Filho \\ Department of Industrial Engineering \\ Federal University of São Carlos \\ moacir@dep.ufscar.br
}

\author{
Elaine Mosconi \\ Business School \\ Université de Sherbrooke \\ elaine.mosconi@usherbrooke.ca
}

\begin{abstract}
Potential solutions emerge with the fourth Industrial Revolution technologies, including the Internet of Things, Artificial Intelligence and Blockchain. The global supply chain requires greater traceability and transparency to ensure product security, efficient management and sustainable performance. However, traditional supply chains face several challenges related to traceability systems. This paper aims to analyse academic literature regarding Blockchain adoption in supply chains to improve traceability and transparency from a sustainable perspective. A systematic literature review was performed in order to provide verifiable evidence; we focused our investigation on empirical and modelling articles. Results suggest that Blockchain benefits occur through three levels: macro-level (overall supply chain network), meso-level (such as dyadic relationships) and micro-level (end consumers). However, we note that the contribution's results have not yet reached a consensus. We have therefore proposed three research insights towards addressing the identified gaps.
\end{abstract}

Keywords: Blockchain, supply chain, sustainability, systematic literature review

\section{Introduction}

Since the United Nations Conference on the Human Environment in Stockholm in 1972, we have witnessed a growing awareness of worldwide sustainability issues [1]. Both professionals and scientists have been placing a strong focus on the sustainability concept. Sustainability studies have frequently focused on practical applications, and they have adopted the Triple Bottom Line perspective, seeking a balance between the three components of sustainability: social, economic, and environmental [2, 3]. In this context, sustainability becomes a key factor in supply chain management. Pressure from the media, local and international institutions, non-governmental organisations (NGOs), consumers and regulations encourage organisations to consider sustainability [4]. These demands have created new opportunities and challenges in current supply chain management, such as the circular economy and carbon tax.

In this context, the flow of today's global and complex products between an increasing number of interacting and geographically distributed stakeholders is characterised by a lack of transparency and trust, which creates uncertainty about product value and origin [5]. Companies use traceability systems to obtain a global and coherent view of their supply chain activities. However, significant challenges still exist in making the collected information reliable and available to each stakeholder. Such information is often difficult to be collected due to technical, financial and social barriers [6].

To overcome these challenges and improve supply chains, organisations embrace emerging technologies such as the Internet of Things (IoT), Artificial Intelligence (AI) and Blockchain. Indeed, Blockchain is a disruptive technology that allows stakeholders to communicate effectively and make supply chains smarter and more sustainable [7]. Studies around Blockchain are strongly influenced by opinion articles and grey literature [8]. They remain mostly exploratory, conceptual, or based on literature reviews, explaining that Blockchain is in its early stages, and access to empirical fieldwork is difficult and limited. These 
contributions seem to conceptually create a buzz around how Blockchain can benefit supply chain goals [9].

This paper, which extends the discussion proposed by Paliwal et al. [10], focuses on empirical evidence regarding Blockchain impacts on supply chain sustainability performance. Our work aims to provide as much evidence as possible about the actual Blockchain's impacts on supply chains from a sustainable perspective. To do so, we conducted a systematic literature review to analyse peer-reviewed articles considering only those adopting empirical contributions and modelling approaches. These articles will provide a more meaningful and detailed understanding of the potential effects of Blockchain technology. We seek to answer the following question: What is the early evidence provided by empirical and modelling studies on Blockchain adoption in the supply chain from a sustainable perspective?

The remainder of this paper is structured as follows: Sections 2 and 3 detail, respectively, the basic concepts and the methodology employed. Section 4 presents the main results, and section 5 discusses some insights. Conclusion and research limitations are presented in Section 6.

\section{Background}

\subsection{Blockchain Technology Overview}

On October 31st, 2008, a white paper entitled "Bitcoin - A Peer-to-Peer Electronic Cash System" was released by Satoshi Nakamoto, which proposed a distributed system, now known as Blockchain, for conducting electronic transactions. It was first materialised as cryptocurrency in early 2009 and, since then, applications of this new technology have broadened with the incorporation of the smart contract and continue to expand beyond finance across a wide range of industries [11].

So far, there are three types of Blockchain. First, public Blockchain is accessible to anyone wishing to interact with the network. It works essentially based on a peer-to-peer protocol, and no organisation has control over the system; rather, each decision is made by consensus among users based on computer code. In fact, Blockchain requires a mechanism to maintain a single version of the transaction history accepted by most participants, called the consensus protocol. Consensus is a central element of every Blockchain. It involves communications among miners to determine the transactions to be included in the database. We find two main consensus Proof of Work (PoW) and Proof of Stake (PoS). For example, Bitcoin and Ethereum use the PoW consensus protocol to verify transactions. Since the latter is computationally intensive and consumes much energy, Ethereum plans to switch to the PoS consensus protocol $[2,12]$.

Second, for confidentiality purposes, a private Blockchain configuration was created, acting in a closed environment where access is limited to previously designated users; an example is MultiChain [13]. A private Blockchain can use PoW or other protocols such as a round-robin protocol to build consensus, where participants add blocks in a fixed order [14]. Finally, the Blockchain consortium (federated) operates with the best features of both types; it is faster and more efficient in terms of confidentiality and verification, such as Hyperledger Fabric [15].

Moreover, smart contracts are computer protocols, and codes stored on a Blockchain that verify data, trigger a payment, create a shipment, or issue an invoice according to predefined contract terms [16]. They can be applied automatically between different participants in the Peer-to-Peer network without the need for a third party. Ethereum and Hyperledger Fabric are two widely used platforms for adopting smart contracts [17]. Blockchain has been widely used for applications beyond finance, such as supply chain management and sustainability. In the following subsection, we introduce the traditional supply chain.

\subsection{Traditional and Sustainable Supply Chain}

Supply chain management includes all processes involved, from product and service development until delivery to end customers. It includes upstream and downstream product flows, financial flows, and information management flow [18]. One of the significant challenges of traditional supply chains is how to track and manage different product operations, both inter-organisation and intra-organisation effectively, to ensure that information is appropriately handled across multiple interacting stakeholders. However, there are several grey areas, mainly due to a lack of reliable data management and traceability systems across the supply chain. These limitations can harm the company's reputation, consumer confidence, and supply chain performance from many perspectives [19].

Moreover, these information gaps influence sustainability and become an essential part of supply chain management. Although widely accepted by researchers and practitioners alike, supply chain sustainability faces difficulties in practical implementations [20]. It stands for a complex concept since managers need to integrate social and environmental dimensions with economic aspects to achieve supply chain sustainability performance, as established by Elkington's (1998) Triple Bottom Line concept [3]. 
Commonly, compliance with sustainable objectives in new and responsible supply chains is monitored by focal companies. They are responsible for any unsustainable behaviour by their suppliers and other supply chain stakeholders. For example, Nike is an organisation that has long been criticised and boycotted for the unethical behaviour of its suppliers in countries where labour markets were less regulated and controlled [21].

In fact, companies have a growing need for tools and strategies to track and trace their products from the first raw material extraction to final product consumption and its return (e.g., recycling or remanufacturing). Currently used processes do not meet these needs because, for example, according to diverse regulations, organisations typically need to ensure traceability only one step forward and one step backwards [5]. In this context, product recall in the event of food contamination may be delayed due to difficulties in identifying the source. Also, the information in central databases can be manipulated easily, i.e., it can be modified or deleted by a malicious user or by an administrator mistake [22]; thus, information suffers from a lack of trustworthiness. In addition to fraud and unreliability, other issues are usually associated with information accuracy, data consistency, information governance and certifications, among others [23, 24]. Many studies turn to the Fourth Industrial Revolution's emerging technologies to improve supply chain traceability, transparency, and sustainability $[5,8,19]$.

\section{Method}

This study follows a systematic literature review protocol, a rigorous and transparent research methodology that aims to reduce errors and bias to identify and evaluate the sample. It guarantees that relevant research is identified, ensures reproducibility and leads to gap identification, controversial issues, and knowledge limits in a structured manner [25]. We followed a three-step approach. First, we collected data from five databases: Science Direct, ABI/Inform, Ebsco, Scopus and Web of Science. Second, we searched these databases using three keywords and associated terms ((Blockchain, "distributed ledger"); (sustain*,"triple bottom line", "green", TBL, "social issue", environ*); ("supply chain", "value chain", "supply network", SCM)). We limited the search to titles, abstracts, and keywords. Finally, we selected only peer-reviewed scientific journal articles. Second, we transferred identified papers to a reference manager software (Zotero) to remove duplicates, obtaining at the end 132 articles. Third, reading titles and abstracts allowed us to keep 83 papers, i.e., only those relevant to our research question.
The identified corpus was examined using thematic analysis. It offers a flexible approach because of its theoretical flexibility that can be adapted to the research needs, providing a rich, detailed and complex data assessment. We let patterns (themes) emerge from the data without relying on a well-defined theoretical framework. Thus, the thematic analysis highlights common threads across the selected articles by assigning codes to the units of meaning, then grouping and organising them into themes and sub-themes [26]. In our context, we proceeded through the following steps: reading the whole paper, generating codes, comparing similarities and differences between the codes, regrouping codes, searching and then reviewing themes. Finally, two major themes were identified, (1) supply chain main issues and (2) Blockchain sustainability benefits presented in sub-sections 4.2 and 4.3 , respectively. To reduce bias during the data categorisation and extraction process, we used a qualitative data analysis software, the MAXQDA 2020.

\section{Results}

\subsection{Classification by design approach}

We first categorised the identified papers according to the scientific research method. The nonempirical studies represented $58 \%$ of our corpus and were excluded from our analysis. For empirical studies, we distinguished qualitative and quantitative studies. Empirical qualitative techniques following a single or multiple case studies approach attained 15\%, mainly using a semi-structured interview and secondary data. Empirical quantitative techniques following a multicriteria approach reached $11 \%$, such as Analytic Hierarchy Process (AHP), Principal Component Analysis (PCA), decision-making trial and evaluation laboratory (DEMATEL) based on a literature review and data collected through panels of experts. Moreover, several research results incorporate modelling (16\%). For example, analytical modelling and simulation were based on theory and abstract data, experiments and prototypes were based on real-world data, or using both simulation and prototypes, i.e., hybrid cases.

In addition to empirical contributions, we decided to keep modelling research since it has become a relevant methodological approach to theory development in the scientific literature [27]. In the following sub-sections, we present the sustainable supply chain issues and discuss Blockchain's adoption benefits.

\subsection{Main Supply Chains Issues and Traditional Practices}

In the reviewed literature, we identified several supply chains challenges such as partial and heterogeneous traceability processes leading to lack of 
transparency; centralised information systems that are vulnerable to malicious threats; lack of interoperability between different companies' IT systems; and supply chain complexity resulting from global outsourcing. The current practices to resolve them are limited, timeconsuming and in some cases costly. We classify our findings into four main categories, as summarised in Table 1.

Table 1 Supply chain main issues and current practices

\begin{tabular}{|c|c|c|c|c|}
\hline & Traceability system & $\begin{array}{l}\text { Information systems } \\
\text { security }\end{array}$ & $\begin{array}{l}\text { Digitalisation and } \\
\text { communication }\end{array}$ & Globalisation \\
\hline Issues & $\begin{array}{l}\text {-Lack of transparency } \\
\text { - Heterogeneous } \\
\text { traceability } \\
\text {-Increasing product } \\
\text { fraud. }\end{array}$ & $\begin{array}{l}\text {-The central information } \\
\text { system is exposed to } \\
\text { errors, hacking, and } \\
\text { attacks. } \\
\text {-Unreliable information. }\end{array}$ & $\begin{array}{l}\text {-Lack of digitalisation } \\
\text { process. } \\
\text {-Communication issues } \\
\text { among multiple } \\
\text { stakeholders. }\end{array}$ & $\begin{array}{l}\text {-High interdependency } \\
\text { and complexity between } \\
\text { organisations. } \\
\text {-Fragmented supply } \\
\text { chain }\end{array}$ \\
\hline $\begin{array}{l}\text { Current } \\
\text { practice }\end{array}$ & $\begin{array}{l}\text {-Use of RFID, QR, and } \\
\text { NFC technologies } \\
\text {-Each company has its } \\
\text { own information storage } \\
\text { system. } \\
\text {-Verification through } \\
\text { audits to ensure } \\
\text { compliance. }\end{array}$ & $\begin{array}{l}\text {-Internal protection } \\
\text { system. } \\
\text {-Using third-party } \\
\text { services to store data. }\end{array}$ & $\begin{array}{l}\text {-ERP system. } \\
\text {-Inter-organisation } \\
\text { communication system. } \\
\text {-Inventory management } \\
\text { system. }\end{array}$ & $\begin{array}{l}\text {-Selection of suppliers } \\
\text { according to ethical } \\
\text { criteria and for long } \\
\text { periods. } \\
\text {-Achieving product } \\
\text { transfer balance through } \\
\text { different transportation } \\
\text { means. }\end{array}$ \\
\hline Limits & $\begin{array}{l}\text {-System incompatibility. } \\
\text { - Security issues } \\
\text {-Audits are costly and } \\
\text { time-consuming. }\end{array}$ & $\begin{array}{l}\text {-Vulnerable data security } \\
\text { and reliability }\end{array}$ & $\begin{array}{l}\text {-Manual tasks } \\
\text {-Several ERP/ } \\
\text { communications/ } \\
\text { inventory management } \\
\text { systems. }\end{array}$ & $\begin{array}{l}\text {-Limited supplier } \\
\text { monitoring and a lack of } \\
\text { trust remains. }\end{array}$ \\
\hline References & $\begin{array}{l}{[28][29][8][30][13][31]} \\
{[32]}\end{array}$ & {$[22][33][23][34][35][2]$} & {$[22][36][13]$} & {$[9][37][38]$} \\
\hline
\end{tabular}

\subsection{Blockchain Sustainability Implementation Benefits}

Blockchain can be a tool for supply chain managers to overcome traditional supply chain issues such as transparency and data reliability and achieve sustainability. We have sorted the identified benefits of Blockchain adoption in the supply chain into three levels. First, a macro-level provides a global view of the supply chain network. It includes multiple parts connected directly or indirectly via non-linear and complex relationships. Second, we have identified a less inclusive level, the meso-level. It focuses more on those parties connected directly, such as dyadic relationships [39]. Finally, we have highlighted the benefits for the end customers who benefit from the final product at the micro-level. Both the meso-level and the micro-level are included in the macro-level.

First, from the macro-level perspective (i.e., the entire supply chain network) presented in Table 2 , the adoption of Blockchain can increase traceability and transparency of the overall supply chain network. The entire structure can be monitored in real-time from the first supplier, like farms, through the production process and logistics to the end consumer using Blockchainbased traceability systems. For example, the SmartDairyTracer, a Blockchain application combined with IoT, Edge Computing and AI, ensures eco-friendly production by tracking animal welfare using connected sensors to improve dairy product traceability [40]. In addition, the startup Wenda combined three technologies, i.e., IoT, Big Data Analytics and Blockchain, to expand traceability in wine supply chains throughout the distribution, conservation, and marketing stages [37]. Other Blockchain applications are worth highlighting, such as the pilot project launched by Provenance in 2016 to improve traceability in the fishing industry by integrating Blockchain and smart labelling. In addition, the Everledger Blockchain platform aims to ensure traceability and authenticity of diamonds, and Walmart's project has successfully reduced the time required to track food from days to seconds [13].

Blockchain initiatives are noticed in several sectors, such as the food and transportation industries. Food supply chains have an additional challenge compared to other industries; they deal with perishable and sensitive materials. Indeed, in an end-to-end connected food supply chain, from farm to end consumer, water consumption, pesticide use, and food transit can be monitored, controlled, and optimised using Blockchain, IoT, and smart contracts. Such an application can reduce energy consumption [40]. Moreover, in the shipping industry, companies facing demand volatility can use Blockchain to provide greater visibility and accuracy in freight risk analysis. Such a highly secure and fast application has shortened processing times, reduced delivery time, and increased ocean shipments, implying that companies efficiently 
optimise space for air transport and decrease costs [9]. Blockchain has also been used to ensure a sustainable ecosystem in the automotive industry to fully incorporate the product life cycle, increasing trust and reducing trade friction between supply chain stakeholders [29].

Indeed, the traditional linear supply chain model gives way to a cyclical model through which recovered products are fed back into the traditional supply chain to build a value loop. Blockchain technology and smart contracts provide reliable data and secure records from raw material to end-of-life product recycling, creating a sustainable ecosystem [29]. In addition, the adoption of Blockchain minimises environmental impact. It facilitates product recovery management and encourages recycling and reuse of used products by providing more transparency of repackaged product life cycle [41].

As counterfeit risk management is essential in several products (wine, fish, automotive, food etc.), industries facing these risks are more likely to adopt Blockchain in their supply chain to protect their products, brands, and customers [13]. Thanks to Blockchain immutability principle, the information can hardly be tampered with by counterfeiters since products origins are certified, and in case of any alteration, the products can be identified and then isolated without compromising the whole supply chain. Indeed, the case study analysis performed by Caldarelli et al. (2020) demonstrated the effectiveness of using Blockchain to prevent counterfeiters beyond local authorities' scope [8].

Moreover, a Blockchain platform is being developed to monitor the entire supply chain and optimise carbon emissions by generating automatic instructions through smart contracts [4]. Reducing carbon emissions is an important factor in making businesses environmentally responsible. The carbon tax consists of charging companies according to their GHG (greenhouse gas) index of emissions. Each company has to comply with the set emission threshold. A Blockchain will monitor and rebalance carbon emissions by triggering smart contracts and enabling it to check the carbon emission limit, apply adjustments, and make environmentally friendly decisions in real-time. It collects and records carbon emissions data based on how they are generated, i.e., during transportation, manufacturing, or storage. In addition, if the company emits more than expected, it pays a tax, and if it emits less than expected, it can trade carbon allowances with other companies. Overall, such a Blockchain initiative can reduce carbon emissions [4].

Table 2 Macro-level Blockchain benefits

\begin{tabular}{|l|l|l|l|}
\hline Blockchain benefits & Evidence & Industry & Ref. \\
\hline $\begin{array}{l}\text { End-to-end traceability and } \\
\text { boosted visibility }\end{array}$ & $\begin{array}{l}\text { Case study/Pilot project } \\
\text { Simulation }\end{array}$ & $\begin{array}{l}\text { NS } \\
\text { Transportation (Port) }\end{array}$ & {$[22,37]$} \\
\hline Recycling promotion & Simulation/ Prototype & Agri-food & {$[29]$} \\
\hline Counterfeiting reduction & $\begin{array}{l}\text { Case study } \\
\text { Simulation/ Prototype }\end{array}$ & Agri-food & {$[8,29]$} \\
\hline Carbon emissions monitoring & Analytical model/experiment & NS & {$[4]$} \\
\hline Waste products reduction & $\begin{array}{l}\text { Case study } \\
\text { Analytical model }\end{array}$ & $\begin{array}{l}\text { Transportation (airport) } \\
\text { Fashion }\end{array}$ & {$[2,42]$} \\
\hline Eco-friendly production & $\begin{array}{l}\text { Simulation } \\
\text { Agri-food } \\
\text { Electronique }\end{array}$ & {$[40,41]$} \\
\hline $\begin{array}{l}\text { Information efficiency } \\
\text { improvement }\end{array}$ & $\begin{array}{l}\text { Sulti-case studies } \\
\text { Simulation/ Prototype }\end{array}$ & $\begin{array}{l}\text { Food-Transport-Diamonds-Fishing } \\
\text { Agri-food }\end{array}$ & {$[13,29]$} \\
\hline Fast access to immutable audit & Case study/Proof of concept & $\begin{array}{l}\text { Transportation (shipping) } \\
\text { Food-Agri-Food- Transport- } \\
\text { Diamonds-Fishing }\end{array}$ & {$[23,43][13$,} \\
\hline Real-time decision-making & $\begin{array}{l}\text { Analytical model/Simulation } \\
\text { Analytical model/Experiment }\end{array}$ & $\begin{array}{l}\text { NS } \\
\text { Transportation (port) }\end{array}$ & {$[4,9]$} \\
\hline Transit time reduction & $\begin{array}{l}\text { Case study/ Experiment } \\
\text { Analytical model/Simulation }\end{array}$ & $\begin{array}{l}\text { Agri-food } \\
\text { Transportation (port) }\end{array}$ & {$[9,40]$} \\
\hline NS=Not Specified & & \\
\hline
\end{tabular}

NS=Not Specified

Second, the meso-level is summarised in Table 3. Through the use of Blockchain, small and medium-size enterprises (SMEs) gain an advantage in a highly competitive environment [45]. It was also confirmed in the studies of Ko et al. (2018) and Philip et al. (2019) that Blockchain implementation is cost-saving for small firms and can support global competition and equity by easing challenges, reducing entry barriers, and weakening dominant players' positions [23,37]. Blockchain implementation allowed more accessible logistic financing for SME e-commerce retailers using smart contracts [47]. Combining Blockchain and smart contracts enables automation and optimisation of operations, reducing manual verification errors and improving working conditions $[36,48]$. 
Table 3 Meso-level Blockchain benefits

\begin{tabular}{|l|l|l|l|}
\hline Blockchain benefits & Evidence & Industry & Ref. \\
\hline Facilitate financing e-commerce retail & Experimental case study/ Prototype & E-commerce & {$[49]$} \\
\hline $\begin{array}{l}\text { Market access challenges and barriers } \\
\text { reduction }\end{array}$ & Case study & Food & {$[36]$} \\
\hline Competitive advantage achievement & $\begin{array}{l}\text { Multi-case study } \\
\text { Expert opinion } \\
\text { Analytical model }\end{array}$ & Food & {$[36,45,46]$} \\
\hline $\begin{array}{l}\text { Sustainable suppliers' selection and } \\
\text { evaluation }\end{array}$ & Simulation & NA & {$[38]$} \\
\hline Inventory management improvement & Case study & Transportation (airport) & {$[2]$} \\
\hline $\begin{array}{l}\text { Manual operations and human error } \\
\text { reduction }\end{array}$ & $\begin{array}{l}\text { Multi-case studies } \\
\text { Simulation/Proof of concept }\end{array}$ & $\begin{array}{l}\text { Healthcare } \\
\text { Food }\end{array}$ & {$[36,48]$} \\
\hline Expediting administrative procedures & $\begin{array}{l}\text { Case study/Pilot study } \\
\text { Multi-case study/Simulation }\end{array}$ & $\begin{array}{l}\text { NA } \\
\text { Food }\end{array}$ & {$[22,36]$} \\
\hline Data quality improvement & $\begin{array}{l}\text { Analytical model } \\
\text { Experimental study }\end{array}$ & $\begin{array}{l}\text { Fashion } \\
\text { E-commerce }\end{array}$ & {$[42,50]$} \\
\hline
\end{tabular}

In addition, Martinez et al. (2019) presented a pilot application developed using the company's internal resources with basic resources and programming skills. Blockchain implementation has improved time processing and workload for order management processes from days to less than one minute by simplifying operations [22]. The use of smart contracts has made it easier to automatically manage the negotiation processes and exclude shipbrokers in the case of port logistics as a central intermediary [36].

Blockchain can support decision-making when evaluating suppliers. A combination of IoT to collect real-time data, Machine Learning to automate the supplier evaluation process, and Blockchain technology to share data securely allow companies to achieve an efficient supplier capability evaluation system by ensuring real-time data sharing and a reliable exchange environment. Indeed, it replaces the traditional, manual and subjective environment by objectively collecting and evaluating suppliers' performance [38].
Third, the micro-level (i.e., end consumers) is presented in Table 4. Most consumers' concerns and uncertainties regarding reconditioned products may be reduced since they will access product data, such as origin and process history, ensuring sustainability, safety, and security [41]. Choi and Luo (2019) research showed that implementing Blockchain improves consumers social welfare [42]. By using distributed applications, consumers build an interactive and continuous relationship with producers. Several studies integrate a front-end interface with the consumers in Blockchain development, such as Wenda for wine supply chain [37], Bitbarista for coffee supply chain and autonomous machine experience [31], and Cydon for data management [23]. Violino et al. (2019) compared consumer preferences about three olive oil traceability systems: Near Field Communication (NFC), Radio Frequency Identification (RFID), and QR code tagbased Blockchain. The third system gained more attention from consumers due to the immutable and traceable information access through Blockchain, the ease of access to information through QR tag, and the use of a gamification strategy and a prize-winning mechanism to build consumers loyalty [28].

Table 4 Micro-level Blockchain benefits

\begin{tabular}{|l|l|l|l|}
\hline Blockchain benefits & Evidence & Industry & Ref. \\
\hline Concern reduction & $\begin{array}{l}\text { Multi-case studies } \\
\text { Experimentation }\end{array}$ & $\begin{array}{l}\text { Food } \\
\text { Transportation (Port) }\end{array}$ & {$[42,47,53]$} \\
\hline Access to reliable information & Case study & $\begin{array}{l}\text { Food - E-commerce } \\
\text { Transport (Port) }\end{array}$ & {$[21,26,42]$} \\
\hline Uncertainty reduction & $\begin{array}{l}\text { Case study/Pilot study } \\
\text { Experimentation }\end{array}$ & $\begin{array}{l}\text { NA - Transportation } \\
\text { (automotive)-Agri-Food }\end{array}$ & {$[32,41,47]$} \\
\hline $\begin{array}{l}\text { Meet consumer satisfaction and } \\
\text { protection }\end{array}$ & Analytical model & Fashion & {$[42]$} \\
\hline $\begin{array}{l}\text { Consumer-production interactive and } \\
\text { continuous relationship }\end{array}$ & Case study & Transportation (Port) & {$[37]$} \\
\hline
\end{tabular}


We found that some studies address that consumers should participate in the expenses resulting from introducing new traceability mechanisms [43]. Survey results revealed that $94 \%$ of consumers are in favour of obtaining more information through the implementation of new technologies and are even willing to pay a higher price (up to 17\%) [28]. Nevertheless, careful consideration must be given to which information should be shared with consumers and how to involve them because overloading them with information may be negatively perceived and unappreciated [31].

While we may tend to see Blockchain being promoted, a step back allows us to identify all the other technologies that support and are combined with Blockchain to achieve direct and indirect benefits. Thus, we identify various emerging technologies from I4.0 that have been integrated with Blockchain, including IoT, such as RFID (Radio Frequency Identification), and barcode variants (e.g., QR), Big Data, AI and Edge Computing. We also note that the industries that are testing and adopting Blockchain are mostly from food/agri-food and transport sectors.

\section{Emerging Research Perspectives}

It is inspiring to find so much empirical evidence and research that highlights the benefits of Blockchain. However, we are cautious that there is a lack of consensuses related to sustainability and economic impact, trust and intermediaries. We interpret these mixed results in the light of the nascent state of the technology, and the considerations are taken into account in considering the different applied research methodologies, industries, and involved stakeholders.

\subsection{The Balance between the Three Pillars of Sustainability}

Despite the importance of achieving a balance between the three pillars of sustainability, we found few studies that address them simultaneously [29, 40, 41, 42]. For example, companies should ensure consumers and partners satisfaction, loyalty, and trust (social sustainability), create a balance between resources and services (economic sustainability), and include considerations such as carbon footprints, food waste, and biodiversity loss (environmental sustainability). We identified through the scientific literature that it is challenging to balance the pillars of sustainability, as the measurement of social and environmental aspects is difficult to quantify, and companies can decide on their own the relative weighting of the three sustainability parameters [51]. Furthermore, companies must measure their performance to achieve their desired organisational goals. Those who fail to integrate them may miss their supply chains greatest potential [52].
Furthermore, we find that most of the reviewed papers indicate that Blockchain will have a positive economic impact [50]. These studies mention that Blockchain implementation improves logistics and supply chain operations efficiency as the technology speeds up data flow transfer between parties. For example, Kshetri (2018) compared 11 case studies and concludes that Blockchain positively contributes to the goals of speed, reliability, risk reduction, flexibility, and cost after long-term deployment [13]. Moreover, Blockchain combined with AI can reduce product transit time and improves inventory management, ultimately reducing costs $[2,35]$. In addition, reducing manual operations speeds up administrative processes and reduces processing time and costs [36].

While most studies find that Blockchain reduces costs and/or create value, others conclude that its adoption requires consumers to be willing to pay additional costs associated with the newly provided information [28]. Moreover, Choi and Luo (2019) argue that Blockchain adoption may harm the profitability of supply chains unless supported by the government [42]. Therefore, it is essential to have further study to investigate financial aspects.

\subsection{Trust}

Trust is a key factor in supply chain management, and most identified research shows the benefits of Blockchain adoption to improve trust. In fact, the Blockchain, through its distributed and immutable architecture, allows all parties in a respective supply chain to access the same information. This potentially reduces communication and data transfer errors, information asymmetries, opportunistic behaviour, and the need for a trusted third party. Thus, with Blockchain, human trust is substituted by computational and consensus rules in the network $[29,35,36,40]$.

However, a general agreement is not achieved. The study conducted by Caldarelli et al. (2020) highlight Blockchain's need for a trustworthy environment to ensure trust among its users, as the data introduced into the Blockchain may be inaccurate, resulting from scanning, labelling and data entry errors. Thus, the credibility of the oracle, the person, object, or channel that introduces information into the Blockchain is crucial in non-financial applications. The solutions used to overcome this trust and data input problem involve using rigorous physical audits to ensure that the physical assets match the records on the Blockchain. Also, it implicates automating the scanning and recording of products using IoT minimises human intervention and, therefore, errors. Finally, it includes designating a trusted third party, such as a certification authority, to control the information entered on the Blockchain [8]. 
In summary, we distinguish two phenomena, the trust necessary for implementing Blockchain technology and the trust gained by Blockchain implementation. Indeed, Blockchain brings trust in a safe environment, leading us to reflect on the limits of Blockchain adoption. More empirical or modelling studies will be valuable to investigate the influence of Blockchain on trust among organisations.

\subsection{The Future of Intermediaries}

Although intermediaries can effectively add value to the supply chain by providing services such as transportation, trust services and consulting services, they lead to a higher product cost and market monopoly. Moreover, intermediaries often cause delays and information biases, leading to undesirable consequences that propagate along the supply chain [8, 36]. By disintermediation, we mean a move towards shorter and less complex supply chains on which intermediaries such as retailers, resellers, and brokers are bypassed or eliminated [53]. Blockchain has strong disruptive potential, and many contributions address its potential to reduce intermediaries $[29,30,36]$.

However, the Blockchain applications suggest that there are different effects on the structure and functions of intermediaries. We identify three cases that may arise. First, intermediaries will struggle to survive in the new environment, resulting in disintermediation. Second, traditional intermediaries may change their role and expertise, leading to reintermediation. Finally, new intermediaries may emerge, which is known as cybermediation [54]. For example, Caldarelli et al. (2020) discuss the need to use an intermediary to ensure the authenticity of the initial data before it is inserted into the Blockchain; thus, reintermediation appears [8]. Another example is the Blockchain application called OpenBazaar, a decentralised marketplace [55]. Thus, this new application of Blockchain does not enable disintermediation but rather cyber-mediation.

The main conclusion we can draw from the current state of contributions is that adopting Blockchain technology does not have a uniform effect on all intermediaries. We may witness partial or total disintermediation, reintermediation or cyber-mediation depending on the new supply chain dynamics influenced by Blockchain adoption. Therefore, future research is needed to analyse the roles and tasks of intermediaries. Indeed, it is worth knowing the basis on which organisations will choose to develop a Blockchain solution with their own resources, internally as in the case studies by Martinez et al. (2019) [22] or to use commercialised solutions and thus introduce new intermediaries [8, 37]. In the end, we present these insights through some research questions in Table 5.

\section{Table 5 research insights}

\begin{tabular}{|c|c|}
\hline \multirow{3}{*}{$\begin{array}{l}\text { Blockchain and } \\
\text { sustainability }\end{array}$} & $\begin{array}{l}\text { 1. How can the introduction of Blockchain promote, support and drive the Triple Bottom } \\
\text { Line principle? }\end{array}$ \\
\hline & $\begin{array}{l}\text { 2. How can the benefits of technologies such as Blockchain be measured from the Triple } \\
\text { Bottom Line perspective? }\end{array}$ \\
\hline & $\begin{array}{l}\text { 3. Under which business environment and conditions will companies expect economic } \\
\text { benefits from Blockchain implementation? }\end{array}$ \\
\hline \multirow{3}{*}{$\begin{array}{l}\text { Blockchain and } \\
\text { intermediaries }\end{array}$} & $\begin{array}{l}\text { 4. What factors lead intermediaries to transform their services or lose their position to new } \\
\text { intermediaries in Blockchain-based supply chains? }\end{array}$ \\
\hline & 5. How will intermediaries transform their services in Blockchain-based supply chains? \\
\hline & 6. Which drivers support disintermediation in Blockchain-based supply chains? \\
\hline \multirow{2}{*}{$\begin{array}{l}\text { Blockchain and } \\
\text { trust }\end{array}$} & 7. How will organisational trust be advanced in a Blockchain-based supply chain? \\
\hline & $\begin{array}{l}\text { 8. How will companies' reputations support service transformation and user confidence in } \\
\text { the Blockchain-based supply chain? }\end{array}$ \\
\hline
\end{tabular}

\section{Conclusion and Study Limitations}

The potential of Blockchain, one of the Fourth Industrial Revolution technologies', may provide good opportunities to attain superior performance. The reviewed papers included case studies, expert opinion, analytical models and simulations. They allowed us to extend our understanding and confirm Blockchain's opportunities to support supply chain managers facing a range of challenges to increase sustainability goals from macro, meso and micro levels.

We acknowledge that our work has some limitations; even using five databases in English, some relevant papers may be omitted. Also, we have chosen 
to analyse only peer-reviewed journal articles, but we recognise that conference publications are also relevant.

Therefore, through this report, we have identified findings that could help both academics and professionals. Since Blockchain is mainly supported by other technologies, such as Big Data, AI, and IoT to collect and process data in real-time, it would be interesting for future works to distinguish between the direct and indirect benefits. We also identified that few papers considered the three sustainability pillars simultaneously. Moreover, papers are not converging to the same conclusion regarding disintermediation and economic gains, which suggests that the research field is in its infancy. Further, a real-world investigation is needed to clarify Blockchain's influence on visibility in supply chains in terms of creating new collaboration, influencing intermediaries' roles, increasing economic benefits, and improving sustainable performance.

\section{References}

[1] Seuring, S.A., "Assessing the rigor of case study research in supply chain management", Supply Chain Management: An International Journal 13(2), 2008, pp. $128-137$.

[2] Di Vaio, A., and L. Varriale, "Blockchain technology in supply chain management for sustainable performance: Evidence from the airport industry", International Journal of Information Management 52, 2020, pp. 2014-2014.

[3] Elkington, J., "Partnerships from cannibals with forks: The triple bottom line of 21 st-century business", Environmental Quality Management 8(1), 1998, pp. 3751.

[4] Manupati, V.K., T. Schoenherr, M. Ramkumar, S.M. Wagner, S.K. Pabba, and R.I.R. Singh, "A blockchainbased approach for a multi-echelon sustainable supply chain", International Journal of Production Research, 2019, pp. NIL_1-NIL_20.

[5] Astill, J., R.A. Dara, M. Campbell, et al., "Transparency in food supply chains: A review of enabling technology solutions", Trends in Food Science \& Technology 91, 2019, pp. 240-247.

[6] Boenish, R., D. Willard, J.P. Kritzer, and K. Reardon, "Fisheries monitoring: Perspectives from the United States", Aquaculture and Fisheries 5(3), 2020, pp. 131138

[7] Saberi, S., M. Kouhizadeh, J. Sarkis, and L. Shen, "Blockchain technology and its relationships to sustainable supply chain management", International Journal of Production Research 57(7), 2019, pp. 2117 2135.

[8] Caldarelli, G., C. Rossignoli, and A. Zardini, "Overcoming the Blockchain Oracle Problem in the Traceability of Non-Fungible Products", Sustainability 12(6), 2020, pp. 2391-2391

[9] Yoon, J., S. Talluri, H. Yildiz, and C. Sheu, "The value of Blockchain technology implementation in international trades under demand volatility risk", International Journal of Production Research 58(7), 2020, pp. 2163-2183.
[10] Paliwal, V., S. Chandra, and S. Sharma, "Blockchain Technology for Sustainable Supply Chain Management: A Systematic Literature Review and a Classification Framework", Sustainability 12(18), 2020, pp. 76387638 .

[11] Lu, Y., "The blockchain: State-of-the-art and research challenges", Journal of Industrial Information Integration 15, 2019, pp. 80-90.

[12] Xie, J., H. Tang, T. Huang, et al., "A Survey of Blockchain Technology Applied to Smart Cities: Research Issues and Challenges", IEEE Communications Surveys and Tutorials 21(3), 2019, pp. 2794-2830.

[13] Kshetri, N., "1 Blockchain's roles in meeting key supply chain management objectives", International Journal of Information Management 39, 2018, pp. 80 89.

[14] Thompson, C., "How does the Blockchain Work? (Part 3)", Medium, 2018. https://medium.com/blockchainreview/what-blockchain-should-we-use-6ba9cca8df22

[15] Mohanta, B.K., D. Jena, S.S. Panda, and S. Sobhanayak, "Blockchain technology: A survey on applications and security privacy Challenges", Internet of Things 8, 2019, pp. 100107.

[16] Cole, R., M. Stevenson, and J. Aitken, "Blockchain technology: implications for operations and supply chain management", Supply Chain Management 24(4), 2019, pp. 469-483.

[17] Huang, Y., Y. Bian, R. Li, J.L. Zhao, and P. Shi, "Smart contract security: A software lifecycle perspective", IEEE Access 7, 2019, pp. 150184-150202.

[18] Dimitriadis, N.I., and S.C.L. Koh, "Information flow and supply chain management in local production networks: the role of people and information systems", Production Planning \& Control 16(6), 2005, pp. 545554.

[19] Venkatesh, V.G., K. Kang, B. Wang, R.Y. Zhong, and A. Zhang, "System architecture for blockchain based transparency of supply chain social sustainability", Robotics and Computer-Integrated Manufacturing 63, 2020, pp. 1896-1896.

[20] Garetti, M., and M. Taisch, "Sustainable manufacturing: trends and research challenges", Production Planning \& Control 23(2-3), 2012, pp. 83104.

[21] The Economist, "Triple bottom line", The Economist, 2009.

https://www.economist.com/news/2009/11/17/triplebottom-line

[22] Martinez, V., M. Zhao, C. Blujdea, X. Han, A. Neely, and P. Albores, "Blockchain-driven customer order management", International Journal of Operations \& Production Management 39(6/7/8), 2019, pp. 993 1022.

[23] Epiphaniou, G., P. Pillai, M. Bottarelli, H. Al-Khateeb, M. Hammoudesh, and C. Maple, "Electronic Regulation of Data Sharing and Processing Using Smart Ledger Technologies for Supply-Chain Security", IEEE Transactions on Engineering Management 67(4), 2020, pp. 1059-1073.

[24] Köhler, S., and M. Pizzol, "Technology assessment of blockchain-based technologies in the food supply chain", Journal of Cleaner Production 269, 2020, pp. 122193.

[25] Tranfield, D., D. Denyer, and P. Smart, "Towards Methodology for Developing Evidence-Informed 
Management Knowledge by Means of Systematic Review", British Journal of Management 14(3), 2003, pp. 207-222.

[26] Braun, V., and V. Clarke, "Using thematic analysis in psychology", Qualitative Research in Psychology 3(2), 2006, pp. 77-101.

[27] Dominguez, R., and S. Cannella, "Insights on MultiAgent Systems Applications for Supply Chain Management", Sustainability 12(5), 2020, pp. 1935.

[28] Violino, S., F. Pallottino, G. Sperandio, et al., "Are the Innovative Electronic Labels for Extra Virgin Olive Oil Sustainable, Traceable, and Accepted by Consumers?", Foods 8(11), 2019, pp. 529-529.

[29] Sharma, P.K., N. Kumar, and J.H. Park, "BlockchainBased Distributed Framework for Automotive Industry in a Smart City", IEEE Transactions on Industrial Informatics 15(7), 2019, pp. 4197-4205.

[30] Kittipanya-ngam, P., and K.H. Tan, "A framework for food supply chain digitalisation: lessons from Thailand", Production Planning \& Control 31(2-3), 2020, pp. $158-172$.

[31] Tallyn, E., L. Pschetz, R. Gianni, C. Speed, and C. Elsden, "Exploring machine autonomy and provenance data in coffee consumption: A field study of bitbarista", Proceedings of the ACM on Human-Computer Interaction 2(CSCW), 2018.

[32] Dwivedi, S.K., R. Amin, and S. Vollala, "Blockchain based secured information sharing protocol in supply chain management system with key distribution mechanism", Journal of Information Security and Applications 54, 2020, pp. 102554.

[33] Wong, L.-W., L.-Y. Leong, J.-J. Hew, G.W.-H. Tan, and K.-B. Ooi, "Time to seize the digital evolution: Adoption of blockchain in operations and supply chain management among Malaysian SMEs", International Journal of Information Management 52, 2020, pp. 1997-1997.

[34] Kombe, C., A. Sam, M. Ally, and A. Finne, "Blockchain Technology in Sub-Saharan Africa: Where does it fit in Healthcare Systems: A case of Tanzania", Journal of Health Informatics in Developing Countries 13(2), 2019, pp. 1-19.

[35] Helo, P., and A.H.M. Shamsuzzoha, "Real-time supply chain-A blockchain architecture for project deliveries", Robotics and Computer-Integrated Manufacturing 63, 2020, pp. 1909-1909.

[36] Philipp, R., G. Prause, and L. Gerlitz, "Blockchain and Smart Contracts for Entrepreneurial Collaboration in Maritime Supply Chains", Transport and Telecommunication 20(4), 2019, pp. 365-378.

[37] Spadoni, R., M. Nanetti, A. Bondanese, and S. Rivaroli, "Innovative solutions for the wine sector: The role of startups", Wine Economics and Policy 8(2), 2019, pp. $165-170$.

[38] Li, Z., H. Guo, A.V. Barenji, W.M. Wang, Y. Guan, and G.Q. Huang, "A sustainable production capability evaluation mechanism based on blockchain, LSTM, analytic hierarchy process for supply chain network", International Journal of Production Research 58(24), 2020, pp. 7399-7419.

[39] Ritter, T., I.F. Wilkinson, and W.J. Johnston, "Managing in complex business networks", Industrial Marketing Management 33(3), 2004, pp. 175-183.

[40] Alonso, R.S., I. Sitton-Candanedo, O. Garcia, J. Prieto, and S. Rodriguez-Gonzalez, "An intelligent Edge-IoT platform for monitoring livestock and crops in a dairy farming scenario", Ad Hoc Networks 98, 2020, pp. 2047-2047.

[41] Tozanli, O., E. Kongar, and S.M. Gupta, "Trade-in-toupgrade as a marketing strategy in disassembly-to-order systems at the edge of blockchain technology", International Journal of Production Research 58(23), 2020, pp. 7183-7200.

[42] Choi, T.-M., and S. Luo, "Data quality challenges for sustainable fashion supply chain operations in emerging markets: Roles of blockchain, government sponsors and environment taxes", Transportation Research Part E: Logistics and Transportation Review 131, 2019, pp. 139-152.

[43] Rogerson, M., and G.C. Parry, "Blockchain: case studies in food supply chain visibility", Supply Chain Management-an International Journal 25(5), 2020, pp. 601-614.

[44] $\mathrm{Xu}, \mathrm{H}$. , "Minimising the ripple effect caused by operational risks in a make-to-order supply chain", International Journal of Physical Distribution \& Logistics Management 50(4), 2020, pp. 381-402.

[45] Nayak, G., A.S. Dhaigude, and Y.P. Pai, “A conceptual model of sustainable supply chain management in small and medium enterprises using blockchain technology", Cogent Economics \& Finance 7(1), 2019, pp. 1.

[46] Ko, T., J. Lee, and D. Ryu, "Blockchain technology and manufacturing industry: Real-time transparency and cost savings", Sustainability (Switzerland) 10(11), 2018.

[47] Li, M., S. Shao, Q. Ye, G. Xu, and G.Q. Huang, "Blockchain-enabled logistics finance execution platform for capital-constrained E-commerce retail", Robotics and Computer-Integrated Manufacturing 65, 2020, pp. 1962-1962.

[48] Figueroa, S., Javier Añorga, and Saioa Arrizabalaga, "An Attribute-Based Access Control Model in RFID Systems Based on Blockchain Decentralized Applications for Healthcare Environments", Computers(3), 2019, pp. 57.

[49] Liu, Z., and Z. Li, "A blockchain-based framework of cross-border e-commerce supply chain”, International Journal of Information Management 52, 2020, pp. 2059-2059.

[50] Kumar, G., R. Saha, W.J. Buchanan, et al., "Decentralised accessibility of e-commerce products through blockchain technology", Sustainable Cities and Society 62, 2020, pp. 2361-2361.

[51] Ahi, P., and C. Searcy, "Assessing sustainability in the supply chain: A triple bottom line approach", Applied Mathematical Modelling 39(10), 2015, pp. 2882-2896.

[52] Gunasekaran, A., C. Patel, and R.E. McGaughey, "A framework for supply chain performance measurement", International Journal of Production Economics 87(3), 2004, pp. 333-347.

[53] Atkinson, R.D., "The Revenge of the Disintermediated", Policy Paper, 2001, pp. 23.

[54] Giaglis, G.M., S. Klein, and R.M. O'Keefe, "The role of intermediaries in electronic marketplaces: developing a contingency model", Information Systems Journal 12(3), 2002, pp. 231-246.

[55] Tönnissen, S., and F. Teuteberg, "Analysing the impact of blockchain-technology for operations and supply chain management: An explanatory model drawn from multiple case studies", International Journal of Information Management 52, 2020, pp. 101953. 